\title{
Three-photon states in nonlinear crystal superlattices
}

\author{
D. A. Antonosyan, ${ }^{1,2, \text {, T. V. Gevorgyan, }}{ }^{2}$ and G. Yu. Kryuchkyan ${ }^{1,2, \text { 团 }}$ \\ ${ }^{1}$ Yerevan State University, Alex Manoogian 1, 0025, Yerevan, Armenia \\ ${ }^{2}$ Institute for Physical Researches, National Academy of Sciences, \\ Ashtarak-2, 0203, Ashtarak, Armenia
}

\begin{abstract}
It has been a longstanding goal in quantum optics to realize controllable sources generating joint multiphoton states, particularly, photon triplet with arbitrary spectral characteristics. We demonstrate that such sources can be realized via cascaded parametric down-conversion (PDC) in superlattice structures of nonlinear and linear segments. We consider scheme that involves two parametric processes: $\omega_{0} \rightarrow \omega_{1}+\omega_{2}, \omega_{2} \rightarrow \omega_{1}+\omega_{1}$ under pulsed pump and investigate spontaneous creation of photon triplet as well as generation of high-intensity mode in intracavity three-photon splitting. We show preparation of Greenberger-Horne-Zeilinger polarization entangled states in cascaded type-II and type-I PDC in framework of consideration dual-grid structure that involves two periodically-poled crystals. We demonstrate the method of compensation of the dispersive effects in non-linear segments by appropriately chosen linear dispersive segments of superlattice for preparation heralded joint states of two polarized photons. In the case of intracavity three-photon splitting, we concentrate on investigation of photon-number distributions, third-order photon-number correlation function as well as the Wigner functions. These quantities are observed both for short interaction time intervals and in over transient regime, when dissipative effects are essential.
\end{abstract}

PACS numbers: 42.65.Lm, 42.50.Dv, 42.65.Yj

\section{INTRODUCTION}

Quantum communication and optical quantum computation rely on controlled preparation of well-defined photonic states as well as entangled states of electromagnetic modes. Generating of these states is a primary task for the application of quantum information processing. Particularly, the experimental preparation, manipulation, and detection of multiphoton states are of great interest for the implementation of quantum communication schemes, quantum gates and for fundamental tests of quantum theory [1-3]. There exist a number of proposal for implementing optical quantum-state engineering (see, for example, reviewed paper [4]). Recently, this program has been realized in details for single-photon and single-mode states. The method of manipulating overall group delay mismatches between interacting in a multilayered structures (superlattice structures) by compensating dispersive effects in nonlinear segments by chosen linear segments have been developed [5] for synthesis of twin photon states. In this way, the demonstration of heralded single photons prepared in pure quantum states from a spontaneous parametric down-conversion (SPDC) has been done in series of papers [8] and engineering of quantum-optical state has been demonstrated in [9]. There are some attempts to expand this strategy for producing heralded two-photon polarization entangled states, since one promising approach to the practical realization many of these tasks relies on qubits that are encoded in the polarization states of single photons.

*antonosyand@ysu.am

† kryuchkyan@ysu.am
It was shown that the production of one heralded polarization entangled photon pair using only conventional down-conversion sources, linear optical elements, and projective measurements requires at least three initial pairs [10]. An experimental scheme for producing heralded two-photon entanglement, which relies on triplepair emission from a single down-conversion source was proposed [11] and experimentally realized [12].

It has an important goal of quantum optics to realize effective sources producing three-photon entangled states. Up to now, several physical systems have been proposed for the direct generation of photon triplet including cascaded spontaneous parametric downconversion [13, 14], quantum dots [15] as well as electronpositron annihilation [16]. Experimentally, three-photon down-conversion was studied in third-order nonlinear media $17-19]$ and also by using cascaded second-order nonlinear parametric processes [20]. Most recently, direct generation of photon triplets using cascaded photon-pairs has been demonstrated in periodically poled lithium niobate (PPNL) crystals 21]. This process will initiate new class of experiments in quantum information technologies using photons. Full characterization of a three-photon Greenberger-Horne-Zeilinger (GHZ) state using quantum state tomography has been performed [22]. Very recently, the distinction of three-photon GHZ and W states entangled in time and space has been reported by comparing the second-order and third-order correlation functions [23]. As an application of this approach a method of generation of a narrowband three-photon $\mathrm{W}$ state entangled in time (or energy) via two four-wave mixing processes in cold atomic gas media has also been proposed and studied [24]. It was also shown that quantum-optical states based on three-photon down-conversion can be effectively generated in cascaded optical parametric oscilla- 
tor (OPO). The cascaded OPO was proposed [25, 26] and experimentally realized by using the dual-grid method of quasi-phase matching (QPM) 32]. This scheme that involves cascading second-order nonlinearities is based on parametric processes of splitting and summing in which the frequencies between the pump and two subharmonics frequencies are in the ratio of $3: 2: 1$. This cascaded configuration is different from the ones proposed in the cited papers. It involves the fundamental mode driven by an external pump field at the frequency $\omega_{0}$ and two subharmonic modes $\omega_{2}=\frac{2 \omega_{0}}{3}$, and $\omega_{1}=\frac{\omega_{0}}{3}$. A remarkable feature of OPO is comparatively low generation threshold [25] in comparison with the scheme of direct intracavity three-photon down-conversion, where the pump power threshold is determined by third-order susceptibility [17]. Another remarkable feature is formation of three phase locked states for both subharmonic modes equally spaced by $\frac{2 \pi}{3}$.

In this paper, we consider semiclassical- and quantumproperties of photon triplets generated in cascaded parametric process noted above: $\omega_{0} \rightarrow \omega_{1}+\omega_{2}$ and $\omega_{2} \rightarrow$ $\omega_{1}+\omega_{1}$. This setup offer a variant of the scheme realized in the experiment 21] and leads to generation of triple photons with approximately equal frequencies. In this way two physical cases will be considered: (a) cascaded spontaneous parametric three-photon splitting in a "superlattice" of nonlinear and anisotropic linear segments pumped by an ultrashort pulse; (b) amplification of these processes in the presence of an optical cavity.

Thus, in the first part of this paper we propose detailed studies of quasi-phase matching in cascaded configurations considering complex nonlinear structures for simultaneously phase matching of two parametric processes in optical lattices. We consider dual-grid structure that involve periodically poled crystals for effective generation of photon triplets as in the experiment [21], as well as we propose the analogical scheme for preparation of the three-photon GHZ polarization entangled states. We also consider dual-grid structures with second-order nonlinear and linear materials for generation of photon triplet with arbitrary joint spectrum. In this way we analyze the production of heralded two-photon polarization entanglement in such superlattice by using the conditional method of detection of auxiliary photons. For this goal the various cases of the spectral factorization of three-photon amplitude are analyzed in details.

The second part of the paper is devoted to studies of cascaded OPO on the base of the above analysis. In the cited papers [25, 27] the general theory of OPO has been developed, however, without any concretization of the effective coupling constants between modes subharmonics that are characterized by the wave-vector mismatches of the parametric processes $\omega_{0} \rightarrow \omega_{1}+\omega_{2}$ and $\omega_{2} \rightarrow \omega_{1}+\omega_{1}$. Here, we consider these points in details. Besides this, the lasing of "three-photon" mode is investigated in transition through the generation threshold. This investigation includes also analysis of quantum statistics of modes i.e. photon number distributions, third-order correlation functions and the Wigner function for short interaction time as well as for over transition regime of OPO, when dissipative effects are included.

The paper is arranged as follows. In Sec. II we derive the Hamiltonian of the system and derive three-photon state through QPM concurrent nonlinearities. In Sec. III we briefly describe three-wave type-II interaction in nonlinear superlattice structures, analyze generation of photon triplet in periodically poled nonlinear crystals (PPNC) and propose the scheme for preparation of GHZstates. In Sec. IV we derive the conditions for spectral factorization of the three-photon amplitude. Section V is devoted to the analysis of cascaded OPO. We present the results on photon-number distributions, third-order correlation function of photon-numbers and the Wigner functions for subharmonic modes of OPO for both short interaction time and steady-state regime.

\section{THREE-PHOTON STATE THROUGH QUASI-PHASE MATCHING CONCURRENT NONLINEARITIES}

In this section, we consider multiple optical interactions in $\chi^{(2)}$ nonlinear media, leading to the simultaneous quasi-phase matching (QPM) of two parametric processes. We investigate in details the cascaded processes of three-photon splitting and summing that lead particularly to the three-photon down-conversion. In the scheme the pump field at the frequency $\omega_{0}$, which converts to the subharmonics at the central frequencies: $\omega_{1}=\frac{\omega_{0}}{3}$ and $\omega_{2}=\frac{2 \omega_{0}}{3}$ throughout two cascaded processes: $\omega_{0} \rightarrow \omega_{1}+\omega_{2}$ and $\omega_{2} \rightarrow \omega_{1}+\omega_{1}$. Note, that a complete description of photon triplet requires that all degrees of freedom associated with the quantized electromagnetic field of the occupied optical modes must be taken into account. The specification of the photons involves their polarization, their wave-vector $k$ and frequency or their spatial and time dependence. The dispersion relationship between $k$ and $\omega$ reduces one degree of freedom, such that dependence from the transverse wavevectors $k_{\perp}$ should be taken in account. In this way, the transverse correlations in tripartite entanglement have been studied in the paper [28]. Complete description in which the source produces three-photon states in different modes entangled in time and space has been done in the cited paper [23]. Analogous multimode calculations for cascading parametric systems in the layered media seem to be complicated and can be carried out only by numerical means. However, the full discussion of all degrees of freedom is beyond the scope of this paper, for the further discussion we assume that the spatial modes of the PDC photon pairs are independently decorrelated. We proceed from the single-mode approximation that selects only a single transverse spatial mode for the purpose of this paper. Thus, we consider collinear, onedimensional configurations focusing on studies of spectral correlations and polarization entanglement. Such spatial 
decorrelation can be achieved, for example, by waveguides [7], in which the photons may be emitted only into specific transversely-confined modes. On the other hand, one-dimensional collinear approach used here is also fully consistent for consideration of cascaded PDC, which generates three-photon states in the presence of an optical cavity. This analysis will be presented in Sec.V. Thus, in the discussed approach the Hamiltonian of three-wave interaction reads as

$$
\begin{gathered}
H(t)=S \sum_{\alpha, \beta, \gamma} \int_{0}^{L} d z \chi_{\alpha, \beta, \gamma}^{(2)}(z) E_{\alpha}(z, t) E_{\beta}(z, t) E_{\gamma}(z, t)= \\
H^{(-)}(t)+H^{(+)}(t),
\end{gathered}
$$

where $\chi_{\alpha, \beta, \gamma}^{(2)}(z)$ is the second-order susceptibility of the one-dimensional medium of length $L$. The electric field operator $E_{\alpha}(z, t)$ is the superposition of the three components corresponding to the pump field $E_{L, \alpha}(z, t)$ and the fields of two subharmonics consisting of positive $E_{j, \alpha}^{(+)}(z, t)$ and negative $E_{j, \alpha}^{(-)}(z, t)$ frequency electric field operators $(j=1,2)$. For simplicity, we also omit polarization states of electromagnetic fields and polarization indexes of nonlinear coefficient. The polarization states of modes will be considered in sections III and IV. In this case, the field operators obey

$$
E_{j}^{(+)}(z, t)=i \int \frac{d \omega}{2 \pi} N_{j}(\omega) a_{j}(\omega) e^{i\left(k_{j}(\omega, z) z-\omega t\right)},
$$

where $a_{j}(\omega),(j=1,2)$ are subharmonic modes operators, while classical pump field reads as

$$
E_{L}^{(+)}(z, t)=i \int \frac{d \omega}{2 \pi} E_{L}(\omega) e^{i\left(k_{L}(\omega, z) z-\omega t\right)}
$$

In these equations, $N_{j}(\omega)=\sqrt{\frac{\pi \hbar \omega}{c \epsilon_{0} n_{j}^{2}(\omega) S}}$ is the normalized factor, where $n_{j}(\omega)$ is the refractive index of the medium at the given frequency, $S$ is the cross section area of the beams, $k_{j}(z, \omega)=\frac{\omega_{j}}{c} n_{j}(z, \omega)$. The fields have frequencies centered on carried frequencies $\omega_{j}$ whose bandwidths $\Delta \omega_{j}$ are narrow in comparison to the central frequencies $\omega_{0}, \omega_{1}=\frac{\omega_{0}}{3}$ and $\omega_{2}=\frac{2 \omega_{0}}{3}$.

Note, that below we consider generation of photon triplet due to cascading $\chi^{(2)}$ processes but not due to direct $\chi^{(3)}$ interaction. In this approach the Hamiltonian (11) is written trough the elementary $\chi^{(2)}$ interactions that correspond to the processes: $\omega_{0} \rightarrow \omega_{1}+\omega_{2}$ and $\omega_{2} \rightarrow \omega_{1}+\omega_{1}$ while three-photon down-conversion $\omega_{0} \rightarrow \omega_{1}+\omega_{1}+\omega_{1}$ is calculated in the standard manner (see, for example, [21, 24, 29]) in the second-order of the perturbation theory on $\chi^{(2)}$, (see, formula (11)). Thus, the Hamiltonian of the system that describes nonlinear $\chi^{(2)}$ interactions under the rotating-wave approximation and for neglecting the dependencies from the transverse wave-vectors is calculated as

$$
H(t)=H_{1}(t)+H_{2}(t),
$$

Here and upwards, we use new denotations for the fields operators: $a_{1}=a, a_{2}=b$. The Hamiltonian depends on the Fourier spectra of the function $\chi^{(2)}(z)$

$$
\begin{aligned}
\zeta\left(\omega, \omega_{1}, \omega_{2}\right) & =\int_{0}^{L} d z \chi^{(2)}(z) e^{i \Delta k_{1}(z) z}, \\
\xi\left(\omega_{1}^{\prime}, \omega_{1}^{\prime \prime}, \omega_{2}\right) & =\int_{0}^{L} d z \chi^{(2)}(z) e^{i \Delta k_{2}(z) z},
\end{aligned}
$$

where the phase mismatch vectors are

$$
\begin{gathered}
\Delta k_{1}(z)=k_{L}\left(\omega_{0}, z\right)-k_{1}\left(\omega_{1}, z\right)-k_{2}\left(\omega_{2}, z\right), \\
\Delta k_{2}(z)=k_{2}\left(\omega_{2}, z\right)-k_{1}\left(\omega_{1}^{\prime}, z\right)-k_{1}\left(\omega_{1}^{\prime}, z\right),
\end{gathered}
$$

$\omega=\omega_{1}+\omega_{1}^{\prime}+\omega_{1}^{\prime \prime}$, and $L$ is the total length of the medium. This Hamiltonian is presented in the general form that includes dispersion of interacting waves. Below it will be concretized for multilayered nonlinear structures with different susceptibilities of nonlinearity and with different refractive indexes, pumped by the pulse laser light beam with Gaussian distribution

$$
E_{L}(\omega)=E_{0} \exp \left(-\frac{\tau_{p}^{2}}{2}\left(\omega-\omega_{0}\right)^{2}\right) .
$$

We apply now Eq.(5) for investigation of three-photon states in the cascaded processes $\omega_{0} \rightarrow \omega_{1}+\omega_{1}+\omega_{1}$. The amplitude of this process can be calculated in the secondorder of the perturbation theory with Hamiltonian (5). The vector state in the second-order of the perturbation theory is described in the Dyson series as

$$
|\psi(t)\rangle=\left(\frac{-i}{\hbar}\right)^{2} \int_{-\infty}^{t} d t^{\prime} \int_{-\infty}^{t^{\prime}} d t^{\prime \prime} H\left(t^{\prime}\right) H\left(t^{\prime \prime}\right)|0\rangle_{a}|0\rangle_{b},
$$

where $|0\rangle_{a}$ and $|0\rangle_{b}$ are vacuum states of the modes at the frequencies $\omega_{1}$ and $\omega_{2}$. By using the expressions (5) we 
calculate the final state of this cascaded setup for large time interval $t \rightarrow \infty$. By using the commutation relations $\left[b(\omega), b^{+}\left(\omega^{\prime}\right)\right]=\left[a(\omega), a^{+}\left(\omega^{\prime}\right)\right]=\delta\left(\omega-\omega^{\prime}\right)$, the result is calculated as

$$
|\psi\rangle=\frac{S^{2}}{2 \hbar^{2}} \int d \omega_{1} d \omega_{1}^{\prime} d \omega_{1}^{\prime \prime} N_{1}\left(\omega_{1}\right) N_{1}\left(\omega_{1}^{\prime}\right) N_{1}\left(\omega_{1}^{\prime \prime}\right) \Phi\left(\omega_{1}, \omega_{1}^{\prime}, \omega_{1}^{\prime \prime}\right)\left|0_{b}, 1(\omega), 1\left(\omega^{\prime}\right) 1\left(\omega^{\prime \prime}\right)\right\rangle
$$

where $|1(\omega)\rangle=a(\omega)|0\rangle$ is a single-mode state, $0_{b}, 1(\omega)$

are the occupation numbers of the modes and

$$
\Phi\left(\omega_{1}, \omega_{1}^{\prime}, \omega_{1}^{\prime \prime}\right)=\frac{i}{\pi} E_{L}\left(\omega_{1}+\omega_{1}^{\prime}+\omega_{1}^{\prime \prime}\right) \int \frac{N_{2}^{2}\left(\omega_{2}\right) \zeta\left(\omega, \omega_{1}, \omega_{2}\right) \xi\left(\omega_{1}^{\prime}, \omega_{1}^{\prime \prime}, \omega_{2}\right)}{\omega_{1}^{\prime}+\omega_{1}^{\prime \prime}-\omega_{2}+i \varepsilon} d \omega_{2},
$$

is the amplitude of three-photons at the frequencies $\omega_{1}, \omega_{1}^{\prime}, \omega_{1}^{\prime \prime},(\varepsilon \rightarrow 0)$. Three-photon down-conversion is controlled by energy conservation between the pump and daughter photons $\omega_{0}=\omega_{1}+\omega_{1}^{\prime}+\omega_{1}^{\prime \prime}$, while the amplitude is determined by the pump spectral amplitude and the phase-matching functions.
For the case of degenerate three-photon downconversion: $\omega_{1} \simeq \omega_{1}^{\prime} \simeq \omega_{1}^{\prime \prime} \simeq \frac{\omega_{0}}{3}$ the maximum of this amplitude is realized in the range of resonance $\omega_{2}=$ $\omega_{1}^{\prime}+\omega_{1}^{\prime \prime}=\frac{2 \omega_{0}}{3}$. In this case the Eq. (13) approximately reduces to the result

$$
\Phi\left(\omega_{1}, \omega_{1}^{\prime}, \omega_{1}^{\prime \prime}\right)=E_{L}(\omega) N_{2}^{2}\left(\omega_{2}\right) \zeta\left(\omega, \omega_{1}, \omega_{1}^{\prime}+\omega_{1}^{\prime \prime}\right) \xi\left(\omega_{1}^{\prime}, \omega_{1}^{\prime \prime}, \omega_{1}^{\prime}+\omega_{1}^{\prime \prime}\right) .
$$

Note, that the approximated result (14) can be directly obtained from Eq.(11) if we use the Teylor type timeordering instead of the Dyson series expansion. The basis of this approximation for the parametric processes has been also discussed in [30, 31]. Below, we represent application of these results for effective generation of photon triplet [21], for preparation of both three-photon GHZ entangled states and the heralded polarization states.

\section{THREE-PHOTON SPLITTING IN COMPOSITE NONLINEAR MEDIA}

In this section we first briefly discuss three-wave typeII interaction in one-dimensional $\chi^{(2)}$ nonlinear composite media, consisting of alternating layers with different coefficients of nonlinearity. In this case, the interaction Hamiltonian (11) can be expressed as the sum of interactions in each layer in terms of the electric fields in $n$th layer, $E_{L n}(z, t), E_{j n}^{(-)}(z, t)$

$$
\begin{array}{r}
H(t)=S \sum_{n} \int_{z_{n}}^{z_{n+1}} d z \chi^{(2)}(z) E_{p n}^{*}(z, t) E_{1 n}^{(-)}(z, t) E_{2 n}^{(-)}(z, t) \\
+ \text { h.c. }, \quad(15)
\end{array}
$$

where $\chi^{(2)}(z)$ is the second-order nonlinearity. In approximation that the effects of refraction from the each layers are small the classical pump field $E_{p n}$ and the positive frequency parts of the fields of the subharmonics: $E_{j n}^{(-)}(z, t)(j=1,2)$ can be expressed as

$$
E_{p n}(z, t)=\int d \omega e^{i \omega t} E_{L n}(\omega) e^{\left(-i k_{0 n}(\omega)\left(z-z_{n}\right)\right)}
$$

$$
E_{j n}^{(-)}(z, t)=\int d \omega e^{i \omega t} A_{j n}^{*}(\omega) e^{-i k_{n}(\omega)\left(z-z_{n}\right)} a_{j}^{+}(\omega) .
$$

The electric fields (16), (17) have been studied in 29] for one dimensional composite materials. In the case of composite layered structure the coupling function: $\xi$ 
(and/or $\zeta$ ), (see Eqs.(6),(7)) could be rewritten in the following form (see, the paper [5])

$$
\begin{array}{r}
\xi=\sum_{n} \int_{z_{n}}^{z_{n+1}} \chi_{n} e^{i \Delta k_{n} z} d z= \\
\sum_{m} l_{m} \chi_{m} e^{-i\left(\varphi_{m}+\frac{\Delta k_{m} l_{m}}{2}\right)} \operatorname{sinc}\left(\frac{\Delta k_{m} l_{m}}{2}\right), \\
\varphi_{m}=\sum_{n}^{m-1} l_{n} \Delta k_{n}, \quad \varphi_{1}=0 .
\end{array}
$$

Here, $\chi_{m}$ is the second-order susceptibility in the $m$-th layer, $l_{m}$ is the length and $\Delta k_{m}$ is the phase mismatch vector for the $m$-th layer.

It is easy to generalize these results on the case when polarization structure of three-wave interaction is important. Note, that in this case we should not include a summing on the polarization indexes in the formula (1), if the phase matching of three-waves interaction should take place in each $m$ th layer only for the concrete combinations of three indexes: $\alpha_{m}, \beta_{m}, \gamma_{m}$. In this case, the tensor of second-order susceptibility $\chi_{\alpha, \beta, \gamma}^{(2)}$ reduced to $\chi_{m}=\chi_{\alpha, \beta, \gamma}^{(2)}$ susceptibility in the $m$ th layer. However, in this case the phase mismatch vector for the $m$ th layer $\Delta k_{m}$ also depends on the indexes $\alpha_{m}, \beta_{m}, \gamma_{m}$. Note, that this result is convenient for realizing various schemes of QPM, in particular, for description of periodically poled nonlinear structures. The technique of quasi-phase-matching (QPM) and the availability of multiple gratings in a single periodically poled nonlinear crystal (PPNC) offer flexibility in achieving multiple interactions, which provide enhanced functionality of nonlinear optical devices.

As has been mentioned in Sec. II the maxima of the three-photon process depend on maxima of coupling constants of the each cascading process. Perfect QPM is the condition for the effective process implementation. Thus, in the subsection A QPM of cascading three-photon process: $\omega_{0} \rightarrow \omega_{1}+\omega_{2}, \omega_{2} \rightarrow \omega_{1}+\omega_{1}$ is considered in the complex nonlinear structures with dual-gratings, while the subsection B is devoted to investigation of frequencyuncorrelated photon triplet and subsection generation, C involves patterns of preparation polarization entangled GHZ states in such structures.

\section{A. QPM for generation photon triplets}

In this subsection we develop the theoretical approach of the cascaded generation of photon triplets, particularly, obtained experimentally in 21. We analyzed so called dual-grid nonlinear crystal in frame of mismatching of two SPDC processes. The crystal consists of two periodically poled nonlinear segments with different periods. First segment involves $M$ domains of the length $l_{1}$, with positive $\chi$ and negative $-\chi$ susceptibilities which are interchanged one to other and refractive index $n_{1}$ (corresponding phase-matching function is $\Delta k_{1}$ ), and the second segment consists of $N$ domains of the length $l_{2}$ again with positive $\chi$ and negative $-\chi$ susceptibilities and refractive index $n_{2}$ (corresponding phasematching function is $\Delta k_{2}$ ). The crystal is pumped by a laser field, thus the first segment become primary downconversion source, and photon pair is created. One of the photons from this pair drives the secondary downconversion process in the second segment, generating a second pair, hence, a photon triplet. Because the photon triplet originates from a single pump photon, the created photons are strongly correlated. The $\omega_{0} \rightarrow \omega_{1}+\omega_{2}$ and $\omega_{2} \rightarrow \omega_{1}+\omega_{1}$ processes has been implemented in the first and the second sections of the crystal, correspondingly. We calculate the coupling constants of parametric processes: $\zeta$ and $\xi$ as a function of the finite number of nonlinear segments. Below, we consider spectral amplitudes of these cascaded processes assuming the frequencies $\omega_{1}$ and $\omega_{2}$ as variable quantities distributed around the central values $\omega_{1}=\frac{\omega_{0}}{3}$ and $\omega_{2}=\frac{2 \omega_{0}}{3}$. The three-photon amplitude $\Phi\left(\omega_{1}, \omega_{1}^{\prime}, \omega_{1}^{\prime \prime}\right)$ from Eq.(14) is proportional to the product of the coupling constants and the pump field amplitude: $E_{L}\left(\omega_{1}+\omega_{1}+\omega_{1}^{\prime \prime}\right)$, which is taken in the form of Gaussian distribution (10). This lattice is arranged in the manner that each of the Fourier functions (6), (7) is determines by one of the family of the segments. The details of calculations based on formula (18) can be found in [29]. We derive the following expression for $\zeta$ in terms of Eq. (18)

$$
\begin{gathered}
\zeta=l_{1} \chi e^{-i \frac{L_{1} \Delta k_{1}}{2}} \operatorname{sinc}\left(\frac{l_{1}}{2} \Delta k_{1}\right) \frac{\sin \frac{M l_{1}}{2}\left(\Delta k_{1}-q_{1}\right)}{\sin \frac{l_{1}}{2}\left(\Delta k_{1}-q_{1}\right)}+ \\
l_{2} \chi e^{-i \frac{L_{2} \Delta k_{1}}{2}} \operatorname{sinc}\left(\frac{l_{2}}{2} \Delta k_{1}\right) \frac{\sin \frac{N l_{2}}{2}\left(\Delta k_{1}-q_{2}\right)}{\sin \frac{l_{2}}{2}\left(\Delta k_{1}-q_{2}\right)}(19)
\end{gathered}
$$

where

$$
\Delta k_{1}=k_{L}\left(\omega_{0}\right)-k_{1}\left(\omega_{1}\right)-k_{2}\left(\omega_{2}\right) .
$$

Here $q_{1}=\frac{2 \pi}{d_{1}}, q_{2}=\frac{2 \pi}{d_{2}}$ are the harmonic grating wave vectors for the first and the second segments, respectively, $d_{1}=2 l_{1}, d_{2}=2 l_{2}, L_{1}=M l_{1}$ and $L_{2}=N l_{2}$ are the total lengths of first and second sections, correspondingly.

We assume that effective $\omega_{0} \rightarrow \omega_{1}+\omega_{2}$ process goes in the first section, so the conditions of the perfect QPM is $\Delta k_{1}=q_{1}$. Correspondingly, it is easy to see that the second term in (19) could be neglected proceeding from the QPM condition we are required.

Let us write the approximation of (19) for $M \gg 1$, $L_{1} \gg l_{1}$ and $\Delta k_{1} \simeq q_{1}$. We represent phase-matching function in following form $\Delta k_{1}=\Delta k_{1}^{(0)}+\delta k_{1}$, where $\Delta k_{1}^{(0)}$ is the 0 -order term of the Taylor series and $\delta k_{1}$ is the function of the high-order terms. We rewrite QPM condition in the following form $\Delta k_{1}^{(0)}=q_{1}$, here is asseverated that the constant term could be compensated by the grating wave vector $q_{1}$. Using this approach and assuming that $l_{1} \delta k_{1}$ is very small and could be neglected we could find that the sinc function in the (19) becomes 
a constant multiplier equal to $\frac{2}{\pi}$. Thus, the final approximated form of the $\zeta$ is represented in following form

$$
\zeta=\frac{2}{\pi} M l_{1} \chi e^{-i \frac{L_{1} \Delta k_{1}}{2}} \operatorname{sinc}\left(\frac{L_{1}}{2}\left(\Delta k_{1}-q_{1}\right)\right) .
$$

This result is transformed to an expression that usually used for PPNC in the standard treatment.

The Fourier function (7) is calculated in the following form

$$
\begin{gathered}
\xi=l_{1} \chi e^{-i \frac{L_{1} \Delta k_{2}}{2}} \operatorname{sinc}\left(\frac{l_{1}}{2} \Delta k_{2}\right) \frac{\sin \frac{M l_{1}}{2}\left(\Delta k_{2}-q_{1}\right)}{\sin \frac{l_{1}}{2}\left(\Delta k_{2}-q_{1}\right)}+ \\
l_{2} \chi e^{-i \frac{L_{2} \Delta k_{2}}{2}} \operatorname{sinc}\left(\frac{l_{2}}{2} \Delta k_{2}\right) \frac{\sin \frac{N l_{2}}{2}\left(\Delta k_{2}-q_{2}\right)}{\sin \frac{l_{2}}{2}\left(\Delta k_{2}-q_{2}\right)}(22)
\end{gathered}
$$

where

$$
\Delta k_{2}=k_{2}\left(\omega_{2}\right)-k_{1}\left(\omega_{1}^{\prime}\right)-k_{1}\left(\omega_{1}^{\prime \prime}\right) .
$$

Thus, we assume that effective $\omega_{2} \rightarrow \omega_{1}^{\prime}+\omega_{1}^{\prime \prime}$ process goes in the second section, so the conditions of the perfect QPM is $\Delta k_{2}=q_{2}$. It is easy to see that in this case the first term in (22) could be neglected proceeding from the QPM condition we required. We could write the approximated form for $\xi$ similar to Eq.(21).

So we calculated the coupling constants for the both SPDC processes and found the QPM conditions for their perfect implementation. The both conditions depend on grating wave vectors, consequently, depend on the periods of the segments, which are easily manageable.

\section{B. Frequency-uncorrelated photon triplet}

In this subsection we shortly discuss generation of frequency-uncorrelated triplets of photons, in analogy with the cascaded scheme realized experimentally in [21].

Thus, we assume that photon frequency-uncorrelated pairs in three-wave cascaded process $\omega \rightarrow \omega_{1}+\omega_{2}$, $\omega_{2} \rightarrow \omega_{1}+\omega_{1}$ can be generated. Note, that in typical SPDC experiments a monochromatic pump beam is used. In this situation, the sum of the frequencies of the signal and idler photons is fixed, thus the frequencies of photons are anticorrelated. A number of techniques have been proposed for creating frequency-correlated SPDC under short laser pulses [8]. This point is discussed in details in Sec. IV for the cascaded scheme. In the frequencyuncorrelated approach, we can omit the frequency dependence in the phase-mismatch functions (6), (7) considering them on the fixed central frequencies $\omega, \omega_{1}$ and $\omega_{2}$ with negligible bandwidths $\Delta \omega \ll \omega, \Delta \omega_{i} \ll \omega_{i}$. In this case the effective Hamiltonian (5) is described in the standard way by discreet creation and annihilation operators: $a^{+}, b^{+}$and $a, b$, respectively. The commutation relations are $\left[a, a^{+}\right]=1,\left[b, b^{+}\right]=1$, but without using continuous-variable operators as in the Hamiltonian (5). For the case when photons are spatially separated the result reads as

$$
\begin{gathered}
H=H_{1}+H_{2}, \\
H_{1}=i \hbar\left(\zeta^{\prime} E_{0} b^{+} a_{1}^{+}-\zeta^{\prime *} E_{0}^{*} b a_{1}\right), \\
H_{2}=i \hbar\left(\xi^{\prime} a_{2}^{+} a_{3}^{+} b-\xi^{\prime}{ }^{*} a_{2} a_{3} b^{+}\right) .
\end{gathered}
$$

Here, the subscripts label the spatial mode of photons at the frequencies $\omega_{1}, E_{0}$ describes the amplitude of driving field, $\zeta^{\prime}$ and $\xi^{\prime}$ are nonlinear coupling constants that correspondingly coincide with $\zeta$ and $\xi$ for fixed frequencies $\omega, \omega_{1}, \omega_{2}$, accurate within normalization multipliers. We suppose the initial state of the cascaded system is a vacuum state $\left|\psi_{0}\right\rangle=|0\rangle_{b}|0\rangle_{1}|0\rangle_{2}|0\rangle_{3}$. Thus, time evolution of the system on the formula (11) leads to the final state in the following form [21]

$$
|\psi(t)\rangle=-\frac{t^{2}}{\hbar^{2}} H_{2} H_{1}|\psi\rangle_{i n}=E_{0} \bar{\zeta} \bar{\xi}\left|0_{b}, 1_{1}, 1_{2} 1_{3}\right\rangle .
$$

Here the parameters $\bar{\zeta}=t \zeta^{\prime}, \bar{\xi}=t \xi^{\prime}$ describing the coupling strength between the interacting fields.

\section{Production of GHZ polarization entangled states}

Below we apply the results obtained for preparation of three-photon GHZ states in the cascaded scheme. It is possible for the case when cascaded processes involve polarized photons. Thus, we modify the above results considering three-wave interaction in the following form $\sum_{\alpha, \beta, \gamma} \chi_{\alpha, \beta, \gamma}^{(2)}(z) E_{\alpha}(z, t) E_{\beta}(z, t) E_{\gamma}(z, t)$ (see, formula (10), where $\alpha, \beta, \gamma$ are the indexes of polarization states.

Including into consideration the polarization states of the photons we assume that the type-II process $\omega_{0} \rightarrow$ $\omega_{1}+\omega_{2}$ create the pair of photons with $a$ vertical $V$ and horizontal $H$ polarizations. If the pump field is oriented at $45^{\circ}$ to the horizontal and vertical axes two processes $\omega_{0} \rightarrow \omega_{1}(V)+\omega_{2}(H)$ and $\omega_{0} \rightarrow \omega_{1}(H)+\omega_{2}(V)$ take place in the first crystal with effective coupling constant $\chi$. The second, type-I crystal is arranged in the manner that the following process: $\omega_{2}(V) \rightarrow \omega_{1}(V)+\omega_{2}(V)$ and $\omega_{2}(H) \rightarrow \omega_{1}(H)+\omega_{2}(H)$ should be realized (coupling constant $k$ ). For simplicity we will restrict our attention considering frequency-uncorrelated three-photon states and assume that the process under photons with $(V)$ and $(H)$ polarizations are described by the equal coupling constants. Thus, we assume that photon pairs in three-wave processes : $\omega_{0} \rightarrow \omega_{1}+\omega_{2}, \omega_{2} \rightarrow \omega_{1}^{\prime}+\omega_{1}^{\prime \prime}$ have correlations on the polarization, but not on the spectral lines. In analogy with Eqs.(24)-(26), we model the sum of the corresponding parametric interactions by the following effective Hamiltonian

$$
\begin{gathered}
H=H_{1}+H_{2}, \\
H_{1}=i \hbar \chi E_{0}\left(a_{1}^{+} b_{2}^{+}+a_{2}^{+} b_{1}^{+}\right)+h . c . \\
H_{2}=i \hbar k\left(b_{1}\left(a_{1}^{+}\right)^{2}+b_{2}\left(a_{2}^{+}\right)^{2}\right)+h . c . .
\end{gathered}
$$


Here, $a_{1}$ and $b_{1}$ are the annihilation operators at vertical polarizations, while the operators $a_{2}$ and $b_{2}$ corresponds to the horizontal polarized photons of the frequencies $\omega_{1}$ and $\omega_{2}$, respectively.

Time-evolution of the vector state of the system is de- scribed by the following formula (11) with the Hamiltonians (28)-(30). Choosing the initial state as a vacuum state $\left|\psi_{i n}\right\rangle=|0\rangle_{a_{1}}|0\rangle_{a_{2}}|0\rangle_{b_{1}}|0\rangle_{b_{2}}$ for all modes we derive the final state during time evaluation in the following form

$$
|\psi(t)\rangle=\left(-\frac{i}{\hbar}\right)^{2} t^{2} H_{2} H_{1}|\psi\rangle_{i n}=\bar{\chi} \bar{k} E_{0}\left[\left(a_{1}^{+}\right)^{2} a_{2}^{+}+\left(a_{2}^{+}\right)^{2} a_{1}^{+}\right]|0\rangle_{a_{1}}|0\rangle_{a_{2}}|0\rangle_{b_{1}}|0\rangle_{b_{2}},
$$

where $\bar{\chi}=\chi t$ and $\bar{k}=k t$ are the coupling constants. We generalize this results by including the subscripts labelled the spatial modes of photons at frequency $\omega_{1}$ (see, Eqs. (25), (26), (27). Then, the prepared three-photon polarization-entangled states can be written as

$$
|\psi(t)\rangle=\bar{\chi} \bar{k} E_{0}(|V\rangle|H\rangle|H\rangle+|H\rangle|V\rangle|V\rangle)|0\rangle_{b_{1}}|0\rangle_{b_{2}}
$$

where the states $|V\rangle=a_{1}^{+}|0\rangle_{a_{1}},|H\rangle=a_{2}^{+}|0\rangle_{a_{2}}$ present the vertical and horizontal polarization states of photons at the frequency $\omega_{1}=\frac{\omega_{0}}{3}$. Thus, we demonstrate that in this cascaded scheme triple photons can constitute the Greenberger-Horne-Zeilinger states of light.

\section{QPM IN DUAL SECTION NONLINEAR CRYSTAL WITH LINEAR ELEMENTS}

In this section we investigate an improvement of above approach based on dual-grid nonlinear crystal for engineering controllable, factorable photon states. We consider multilayered structure of second-order nonlinear and linear materials. The key of this investigation is the idea of manipulating overall group velocity delay mismatches between the various fields in structured media for engineering twin-photon states with special temporal and spectral characteristics from. This approach is expanded here for the case of multistep parametric processes SPDC [29]. We analyze the dual-grid structures to mismatch two processes. This method has been recently used in arranging of cascaded OPO 32]. The modified crystal consists of two family of segments: $M$ segments of the length $l_{1}$, with positive $\chi$ and negative $-\chi$ susceptibilities (corresponding phase-matching function is $\Delta k_{1}$ ), and the second is the structure, which consists of $\frac{N}{2}$ segments of the length $l_{2}$ with $\chi$ susceptibilities and $\frac{N}{2}$ linear optical spacers of the length $l_{3}$ (corresponding two phase-matching functions are $\Delta k_{2}$ and $\Delta \kappa$ for the nonlinear and the linear segments). The $\omega_{0} \rightarrow \omega_{1}+\omega_{2}$ and $\omega_{2} \rightarrow \omega_{1}+\omega_{1}$ processes have been implemented in the first and the second sections of the crystal, correspondingly (see, Fig. 1).

We calculate the coupling constants of parametric processes: $\zeta$ and $\xi$ as a function of the finite number of

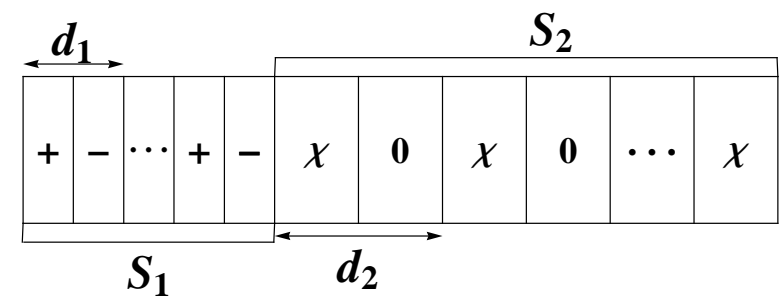

FIG. 1: Scheme of dual section layered structure, $S_{1}$ is a first section with the length $L_{1}$, which involves nonlinear domains of length $l_{1}$ with $\chi_{+}>0$ and $\chi_{-}<0$ (with period $d_{1}$ ) and $S_{2}$ is the second section with the length $L_{2}$, that involves both nonlinear layers of length $l_{2}$ with $\chi$ susceptibilities of the same sign and linear dispersive optical spacers of length $l_{3}$ (period $\left.d_{2}\right)$.

nonlinear and linear segments. We again assume that effective $\omega_{0} \rightarrow \omega_{1}+\omega_{2}$ process goes in the first section, so the conditions of the perfect QPM is $\Delta k_{1}=q\left(q=\frac{2 \pi}{d_{1}}\right)$. Thus, the result for $\zeta$ is exactly the same as the first term in (19). form

The Fourier function (7) is calculated in the following

$$
\xi=l_{2} \chi e^{-i \phi_{2}} \operatorname{sinc}\left(\frac{l_{2}}{2} \Delta k_{2}\right) \frac{\sin \left(\frac{N\left(l_{2}+l_{3}\right) \Delta K}{4}\right)}{\sin \left(\frac{\left(l_{2}+l_{3}\right) \Delta K}{4}\right)},
$$

where $d_{2}=\left(l_{2}+l_{3}\right)$ is a period of the second section, $\phi_{2}=\frac{1}{2} l_{2} \Delta k_{2}+\Delta K\left(l_{2}+l_{3}\right)\left(\frac{N-1}{2}\right)$ and $L_{2}=\frac{N}{2}\left(l_{2}+l_{3}\right)$ is the total length of the second section, where goes $\omega_{2} \rightarrow \omega_{1}+\omega_{1}$ process with the following phase-matching functions (8)

$$
\begin{array}{r}
\Delta k_{2}=k_{2}\left(\omega_{2}\right)-k_{1}\left(\omega_{1}^{\prime}\right)-k_{1}\left(\omega_{1}^{\prime \prime}\right), \\
\Delta \kappa_{2}=\kappa_{2}\left(\omega_{2}\right)-\kappa_{1}\left(\omega_{1}^{\prime}\right)-\kappa_{1}\left(\omega_{1}^{\prime \prime}\right), \\
\Delta K=\overline{l_{2}} \Delta k_{2}+\overline{l_{3}} \Delta \kappa_{2},
\end{array}
$$

where $\overline{l_{i}}=\frac{l_{i}}{l_{2}+l_{3}},(i=2,3), \overline{l_{2}}+\overline{l_{3}}=1$. The condition of perfect QPM in this configuration is $\Delta K=0$. We represent the phase-matching functions of nonlinear: $\Delta k_{2}$ and linear: $\Delta \kappa$ segments as following $\Delta k_{2}=\Delta k_{2}^{(0)}+\delta k_{2}$ and $\Delta \kappa_{2}=\Delta \kappa_{2}^{(0)}+\delta \kappa_{2}$. We assume that each domain 
is aligned such that zero-order constant of nonlinear and linear terms compensate each other $l_{2} \Delta k_{2}^{(0)}+l_{3} \Delta \kappa_{2}^{(0)}=$ 0 . The condition of perfect QPM requires the quantities $\delta k_{2}$ and $\delta \kappa$ exhibit opposite signs: $\bar{l}_{2} \delta k_{2}=-\bar{l}_{3} \delta \kappa$. We additionally require the following condition $\Delta \kappa_{2}^{(0)}=\frac{\pi}{l_{3}}$ for zero-order therm of phase-matching function of linear segments. These conditions are realistic and easily satisfying by managing the lengths of the nonlinear and linear segments, and managing the dispersive parameters of linear segments during crystal growth. The perfect QPM of both processes brings to maxima of coupling constants of the corresponding processes which leads to excellent and effective three-photon down-conversion.

\section{A. Factorable three-photon states. Heralded two-photon polarization states}

Pure single photon states are probably the most fundamental entities in quantum optics, and constitute the starting point for many optically-based quantum enhanced technologies. A basic requirement for many key applications is the ability to generate reliably pure single photon wavepackets capable of high-visibility interference. As we noted, single photon wavepackets may be generated from number-correlated pairs by conditional state preparation. For example, photon pairs produced by the process of spontaneous parametric downconversion (PDC) in a $\chi^{(2)}$ nonlinear medium allow conditional preparation of spectrally factorized photon pairs. The generated pure photons remain polarization entangled, thus, the detection of one photon in the pair heralds the presence of the conjugate photon.

Below we are going to analyze deduced results for the three-photon amplitude of down-converted light for the cascaded processes $\omega_{0} \rightarrow \omega_{2}+\omega_{1}$ and $\omega_{2} \rightarrow \omega_{1}^{\prime}+\omega_{1}^{\prime \prime}$ in the dual-section crystal (see, Fig. 1) and find conditions for generation spectrally factorized photon states. Each of the generated modes has own polarization and we take into account their polarizations in the coupling constants $\xi$ and $\varsigma$ and hence in the amplitude $\Phi\left(\omega_{1}, \omega_{1}^{\prime}, \omega_{1}^{\prime \prime}\right)$ according to the remark presented after formula (18). Thus, we take into account the polarization indexes $\alpha, \beta, \gamma=o, e$ in the vector waves of the modes. To find the conditions of factorization we need to write the three-photon amplitude from Eq.(14) in the Gaussian form. For this reason we expand all phase mismatching vectors in Taylor series and take only the zero- and first-order terms.

$$
l_{1} \Delta k_{1}=l_{1} \Delta k_{1}^{(0)}+T_{1, \alpha} \nu_{1}+T_{2, \beta} \nu_{2}+T_{3, \gamma} \nu_{3},
$$

$$
\begin{gathered}
l_{2} \Delta k_{2}=l_{2} \Delta k_{2}^{(0)}+t_{2, \beta} \nu_{2}+t_{3, \gamma} \nu_{3}, \\
l_{3} \Delta \kappa=l_{3} \Delta \kappa_{2}^{(0)}+\varrho_{2, \beta} \nu_{2}+\varrho_{3, \gamma} \nu_{3} .
\end{gathered}
$$

Here: $\nu_{0}=\omega_{0}, \nu_{1}=\omega_{1}-\frac{\omega_{0}}{3}, \nu_{2}=\omega_{1}^{\prime}-\frac{\omega_{0}}{3}, \nu_{3}=\omega_{1}^{\prime \prime}-\frac{\omega_{0}}{3}$, $\nu=\omega_{2}-\frac{\omega_{0}}{3}$. The coefficients presented above have the following definitions:

$$
\begin{array}{r}
T_{i, \alpha}=l_{1}\left(\left.\frac{d k_{L}\left(\nu_{0}, \beta\right)}{d \nu_{0}}\right|_{\nu_{0}=\omega_{0}}-\left.\frac{d k_{1}\left(\nu_{i}, \alpha\right)}{d \nu_{i}}\right|_{\nu_{i}=0}\right)= \\
l_{1}\left(u_{L, \beta}^{-1}-u_{i, \alpha}^{-1}\right),
\end{array}
$$

where $i=1,2,3, \alpha, \beta=o, e$, for the arbitrary polarization of the laser field;

$$
\begin{array}{r}
t_{\mu, \gamma}=l_{2}\left(\left.\frac{d k_{2}(\nu, \beta)}{d \nu}\right|_{\nu=\frac{\omega_{0}}{3}}-\left.\frac{d k_{1}\left(\nu_{\mu}, \gamma\right)}{d \nu_{\mu}}\right|_{\nu_{\mu}=0}\right)= \\
l_{2}\left(u_{\beta}^{-1}-u_{\mu, \gamma}^{-1}\right),
\end{array}
$$

for arbitrary $\beta=o, e$ polarization of the mode with the central frequency $\frac{2 \omega_{0}}{3}$ in the nonlinear domains;

$$
\begin{array}{r}
\varrho_{\mu, \gamma}=l_{3}\left(\left.\frac{d \kappa_{2}(\nu, \beta)}{d \nu}\right|_{\nu=\frac{\omega_{0}}{3}}-\left.\frac{d \kappa_{1}\left(\nu_{\mu}, \gamma\right)}{d \nu_{\mu}}\right|_{\nu_{\mu}=0}\right)= \\
l_{3}\left(v_{\beta}^{-1}-v_{\mu, \gamma}^{-1}\right),
\end{array}
$$

where $\mu=2,3, \beta, \gamma=o, e$, the $\beta$ polarization of the mode with the central frequency $\frac{2 \omega_{0}}{3}$ in the linear domains is arbitrary.

Here, $T_{i, \alpha}$ are the temporal walkoffs between pumped and down-converted pulses (with the corresponding group velocities $u_{L, \beta}^{-1}$ and $\left.u_{\mu, \alpha}^{-1}\right) ; t_{\mu, \gamma}$ and $\varrho_{\mu, \gamma}$ are the temporal walkoffs between modes with the central frequency $\omega_{2}$ and down-converted pulses with the cental frequency $\omega_{1}$ for nonlinear and linear domains, correspondingly (with the group velocities in nonlinear $u_{\beta}^{-1}, u_{\mu, \gamma}^{-1}$ and linear $v_{\beta}^{-1}, v_{\mu, \gamma}^{-1}$ domains. Here $u_{\beta}^{-1}, v_{\beta}^{-1}$ and $u_{\mu, \gamma}^{-1}, v_{\mu, \gamma}^{-1}$ are the group velocities of modes at frequencies $\omega_{2}$ and $\omega_{1}$, respectively).

Taking into account that the pump pulse has Gaussian form (10) and using the formulas (19), (33) and (38)-(40) we find the following form for the three-photon amplitude

$$
\Phi_{\alpha, \beta, \gamma}\left(\nu_{1}, \nu_{2}, \nu_{3}\right)=F_{\alpha, \beta, \gamma}\left(\nu_{1}, \nu_{2}, \nu_{3}\right) f_{\alpha}\left(\nu_{1}\right) f_{\beta}\left(\nu_{2}\right) f_{\gamma}\left(\nu_{3}\right) \Phi_{\alpha, \beta}\left(\nu_{1}, \nu_{2}\right) \Phi_{\alpha, \gamma}\left(\nu_{1}, \nu_{3}\right) \Phi_{\beta, \gamma}\left(\nu_{2}, \nu_{3}\right),
$$




$$
F_{\alpha, \beta, \gamma}\left(\nu_{1}, \nu_{2}, \nu_{3}\right)=\exp \left(-\frac{i M}{2}\left(T_{1, \alpha} \nu_{1}+T_{2, \beta} \nu_{2}+T_{3, \gamma} \nu_{3}\right)\right) \exp \left(-\frac{i N}{4}\left(t_{2, \beta} \nu_{2}+t_{3, \gamma} \nu_{3}+\varrho_{2, \beta} \nu_{2}+\varrho_{3, \gamma} \nu_{3}\right)\right)
$$

$$
\begin{gathered}
f_{\alpha}\left(\nu_{1}\right)=\exp \left(-\left(\frac{\tau_{p}^{2}}{2}+\frac{M^{2}}{20} T_{1, \alpha}^{2}\right) \nu_{1}^{2}\right) \\
f_{\beta}\left(\nu_{2}\right)=\exp \left(-\left(\frac{\tau_{p}^{2}}{2}+\frac{M^{2}}{20} T_{2, \beta}^{2}+\frac{N^{2}}{80}\left(t_{2, \beta}+\varrho_{2, \beta}\right)^{2}\right) \nu_{2}^{2}\right) \\
f_{\gamma}\left(\nu_{3}\right)=\exp \left(-\left(\frac{\tau_{p}^{2}}{2}+\frac{M^{2}}{20} T_{3, \gamma}^{2}+\frac{N^{2}}{80}\left(t_{3, \gamma}+\varrho_{3, \gamma}\right)^{2}\right) \nu_{3}^{2}\right), \\
\Phi_{\alpha, \beta}\left(\nu_{1}, \nu_{2}\right)=\exp \left(-\left[\tau_{p}^{2}+\frac{M^{2}}{10} T_{1, \alpha} T_{2, \beta}\right] \nu_{1} \nu_{2}\right), \\
\Phi_{\alpha, \gamma}\left(\nu_{1}, \nu_{3}\right)=\exp \left(-\left[\tau_{p}^{2}+\frac{M^{2}}{10} T_{1, \alpha} T_{3, \gamma}\right] \nu_{1} \nu_{3}\right), \\
\Phi_{\beta, \gamma}\left(\nu_{2}, \nu_{3}\right)=\exp \left(-\left[\tau_{p}^{2}+\frac{M^{2}+1}{10} T_{2, \alpha} T_{3, \beta}+\frac{N^{2}}{40}\left(t_{2, \beta}+\varrho_{2, \beta}\right)\left(t_{3, \gamma}+\varrho_{3, \gamma}\right)\right] \nu_{2} \nu_{3}\right) .
\end{gathered}
$$

Let us specify the three-photon process of interest and write conditions for spectral factorization. We concentrate on the discussion of eoo state. It means that in the first cascaded process: $\omega_{0} \rightarrow \omega_{2}+\omega_{1}$ photon with central frequency $\frac{\omega_{0}}{3}$ and polarization $e$ is generated, while in the second process: $\omega_{2} \rightarrow \omega_{1}^{\prime}+\omega_{1}^{\prime \prime}$ two photons with central frequencies $\frac{\omega_{0}}{3}$ and polarizations $o$ are generated. All three photons are strongly correlated, as they are generated from one input photon. Thus, the photon with polarization $e$ can be used as a heralded photon. The condition for generation of correlated photon pairs and the heralded photon is

$$
\left|\Phi_{e, o, o}\left(\nu_{1}, \nu_{2}, \nu_{3}\right)\right|^{2}=\left|\Phi_{e}^{(1)}\left(\nu_{1}\right)\right|^{2}\left|\Phi_{o, o}^{(2)}\left(\nu_{2}, \nu_{3}\right)\right|^{2},
$$

where the separate factor $\left|\Phi_{e}^{(1)}\left(\nu_{1}\right)\right|^{2}=\left|f_{e}\left(\nu_{1}\right)\right|^{2}$ describes one-photon probability and $\left|\Phi_{o,, o}^{(2)}\left(\nu_{2}, \nu_{3}\right)\right|^{2}=$ $\left|f_{o}\left(\nu_{2}\right) \Phi_{o, o}\left(\nu_{2}, \nu_{3}\right) f_{o}\left(\nu_{3}\right)\right|^{2}$ describes two-photon probability.

Here are represented the conditions which have to be satisfied for generation of heralded photon

$$
\begin{gathered}
\tau_{p}^{2}+\frac{M^{2}}{10} T_{1, e} T_{2, o}=0, \\
\tau_{p}^{2}+\frac{M^{2}}{10} T_{1, e} T_{3, o}=0 .
\end{gathered}
$$

These conditions are reduced to one because as it is seen from Eq. (38) $T_{2, o}=T_{3, o}$. We found these conditions from the idea that the joint three-photon state in the Gaussian form (41) must be free of correlations between $\nu_{1}, \nu_{2}$ and $\nu_{1}, \nu_{3}$ spectral components.

Note, that each of conditions (50) in form coincides with the condition derived for the spectral factorization of two-photon amplitude in down-conversion [8, 33]. It is seen from Eq.(38), that the explanation of this results reveals, that this condition may be satisfied either when $u_{2, o}^{-1}<u_{L \beta}^{-1}<u_{1, e}^{-1}$ or when $u_{1, e}^{-1}<u_{L, \beta}^{-1}<u_{2, o}^{-1}$, (for arbitrary polarization of the laser field $\beta$ ). It means that the group velocity of pump pulse ought to lie between the group velocities of the generated pulses with different polarizations. This method provides the optimal compensation for the walkoff effect [33, 34]. It should be point out that it is impossible for type-I down conversion, since in this process photons with similar polarization are produced, consequently, with the same group velocities. We could manage the duration of the pump field $\tau_{p}$ and the length of the domain $l_{1}$ for perfect compensation of the terms in the conditions.

In the result of cascaded three-photon down conversion we could generate completely spectrally factorized pure tree photon states: $\left|\Phi_{e, o, o}\left(\nu_{1}, \nu_{2}, \nu_{3}\right)\right|^{2}=$ $\left|\Phi_{e}^{(1)}\left(\nu_{1}\right)\right|^{2}\left|\Phi_{o}^{(1)}\left(\nu_{2}\right)\right|^{2}\left|\Phi_{o}^{(1)}\left(\nu_{3}\right)\right|^{2}$. The conditions we have 
to satisfy are Eqs.(50) complemented by the following condition

$$
\tau_{p}^{2}+\frac{M^{2}}{10} T_{2, e} T_{3, o}+\frac{N^{2}}{40}\left(t_{2, o}+\varrho_{2, o}\right)\left(t_{3, o}+\varrho_{3, o}\right)=0 .
$$

The last is found from the requirement that the joint three-photon state additionally must be free of the correlations between $\nu_{2}, \nu_{3}$ spectral components. We have observed the dual-section composite crystal with linear elements, which helped us to achieve perfect quasi-phasematching for two simultaneous processes. The linear elements play additional positive role in the compensation of the terms in Eq.(51), thus, in the factorization of the states. In such configurations, there are several parameters which can be managed easily: lengths of the nonlinear $l_{1}, l_{2}$ and linear $l_{3}$ domains, number of linear and nonlinear domains with different lengths, the bandwidth of the pump pulse $\tau_{p}$, as well as the dispersion coefficients of linear domains $\varrho_{\mu}$. The condition (51) is easy achievable, if the dispersion coefficients of the fields in the linear spacers $\varrho_{\mu}$ have the opposite sign to those of in the nonlinear domains $t_{\mu}$. Thus, we could arrange the lengths of nonlinear and linear domains in the second segment, as well as the numbers of domains such that the above condition (51) is fulfilled.

Thus, we have demonstrated the modification of QPM with linear compensator [5, 8] for the cascaded processes. It is shown that this scheme enables the engineering triplet of photons with desirable spectral properties. Using the feature of polarization entanglement, it is possible to consider one of the photons as heralded photon and operate with two renaming states definitely knowing their polarizations.

\section{THREE-PHOTON CORRELATION AND WIGNER FUNCTIONS IN CASCADED OPO}

In the presence of the optical cavity the cascaded threephoton splitting displays new properties that are the subject of this section. We assume that the cavity supports three modes at frequencies $\omega_{0}, \omega_{1}=\frac{\omega_{0}}{3}$ and $\omega_{2}=\frac{2 \omega_{0}}{3}$. The mode $\omega_{0}$ is the pump mode, steerable by an external coherent driving field at the same frequency $\omega_{0}$, while the modes $\omega_{1}$ and $\omega_{2}$ are the modes of the generating subharmonics. In this case, the cavity modes are described by discreet creation and annihilation operators $a^{+}, b^{+}$and $a, b$ as in the Sec. II, III.

The model Hamiltonian for the system, in the rotatingwave approximation, is given by

$$
\begin{array}{r}
H_{i n t}=i \hbar\left(E e^{-i \omega_{0} t} a_{0}^{+}-E^{*} e^{i \omega_{0} t} a_{0}\right)+ \\
i \hbar \zeta^{\prime}\left(a_{0} a^{+} b^{+}-a_{0}^{+} a b\right)+i \hbar \xi^{\prime}\left(a^{+2} b-a^{2} b^{+}\right) .
\end{array}
$$

Here, $E$ describes the amplitude of the driving fields, $\zeta^{\prime}$ and $\xi^{\prime}$ are the nonlinear coupling constants that are proportional to the functions $\zeta$ and $\xi$ (6), (7), correspondingly. In difference from the Hamiltonian (24) this one includes the first term that describes excitation of the pump mode $\omega_{0}$, by a classical pump field at the same frequency.

The cascaded OPO is dissipative, because the modes suffer from losses due to partially transmission of light through the mirrors of the cavity. The master equation for the density operator $\rho$ of the cavity modes in the Limbland form reads as

$$
\frac{\partial \rho}{\partial t}=\frac{1}{i \hbar}\left[H_{i n t} \rho\right]+\sum_{i=0,1,2} \gamma_{i}\left(2 a_{i} \rho a_{i}^{+}-a_{i}^{+} a_{i}-\rho a_{i}^{+} a_{i}\right),
$$

where $\gamma_{i}$ are the cavity damping rates for the modes $\omega_{i}$, $(i=0,1,2)$.

Below, we briefly review the semiclassical results on the model following the papers [25, 27]. For the sake of simplicity let us first of all consider the case of high cavity losses for the fundamental mode $\gamma_{0} \gg \gamma_{1}, \gamma_{2}$, assuming the pump depletion and, hence, its back influence on the modes (1) and (2) are completely neglected. Then the steady state solutions, for $t \gg \gamma_{1}^{-1}, \gamma_{2}^{-1}$, is founded in above threshold operational regime $\varepsilon=\frac{E}{E_{t h}}>1$, where $E_{t h}=\frac{2 \gamma_{0}}{3 \zeta^{\prime}} \sqrt{2 \gamma_{1} \gamma_{2}}$. The mean photon numbers of the modes are

$$
\begin{gathered}
n_{1}=\frac{\gamma_{1} \gamma_{2}}{18 \xi^{\prime 2}}\left(\varepsilon+3 \sqrt{\varepsilon^{2}-1}\right)^{2}, \\
n_{2}=\frac{\gamma_{1}^{2}}{36 \xi^{\prime 2}}\left(\frac{\varepsilon+3 \sqrt{\varepsilon^{2}-1}}{\varepsilon+\sqrt{\varepsilon^{2}-1}}\right)^{2},
\end{gathered}
$$

and the phases are equal to

$$
\phi_{1}=\frac{\Phi}{3}+\frac{2 \pi}{3} n, \quad \phi_{2}=\frac{\Phi}{3}-\frac{2 \pi}{3} n, \quad n=0,1,2,
$$

where $\Phi$ is the phase of the driving field $E=|E| e^{i \Phi}$.

The stability analysis shows, that solutions (54), (55) for both subharmonic modes are stable in the range $E>$ $E_{t h}$. However, a bistable hysteresis-cycle behavior of the $n_{1}$ and $n_{2}$, in dependence on the amplitude $E$, is realized in a small interval $1<\varepsilon<\frac{3}{2 \sqrt{2}}$. Note, that analysis of semiclassical solutions involving the depletion effects has been done in [27].

The second part of this section is devoted to the study of quantum statistical properties of high-intensity subharmonic mode $\omega_{1}$ including calculations of the corresponding Wigner functions and correlation function. Calculations are performed on the framework of the quantum trajectory simulation method [35]. Some details of quantum states diffusion method (QSD), in application to study nonlinear processes in a cavity, can be found in the papers [36, 37].

\section{A. Photon-number distributions and Wigner functions for photon triplets.}

It seems that the correlation between photons in the triplet, as well as quantum interference effects can be 

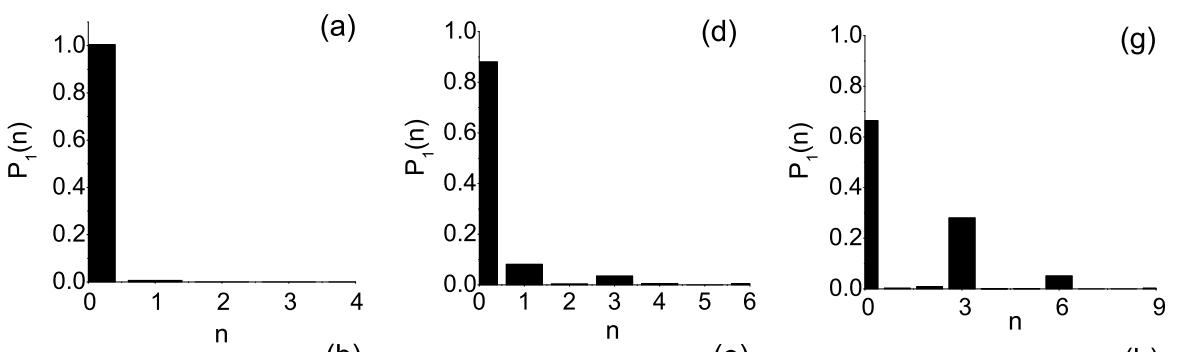

(g)

(b)

(e)
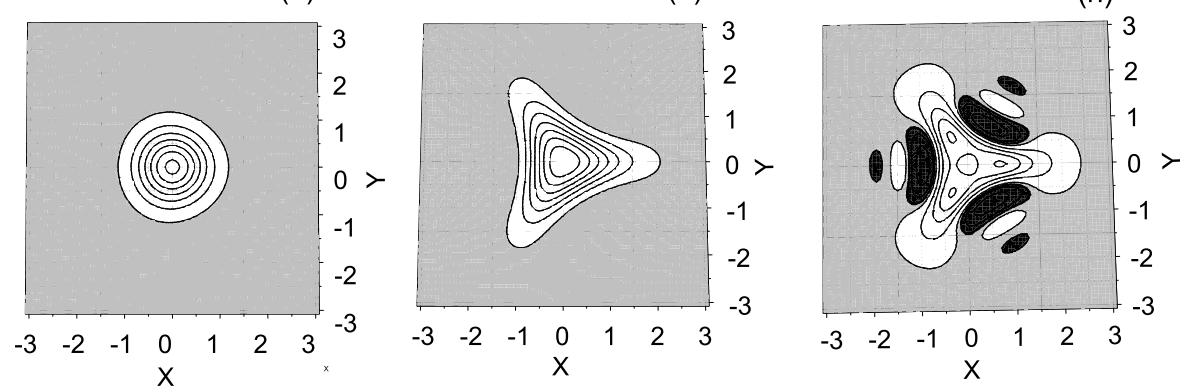

(h)
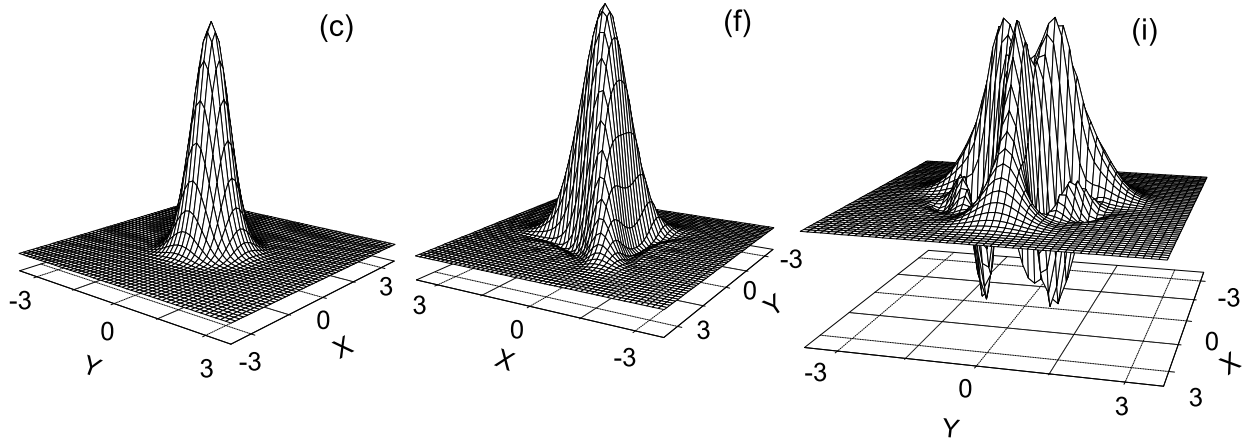

FIG. 2: Photon number distributions (a, d, g), the Wigner functions (c, f, i) and its contour plots (b, e, h) for $\omega_{1}$-mode and for different time intervals, $t=5 \times 10^{-4} \gamma^{-1}$ (a, b, c); $t=5 \times 10^{-3} \gamma^{-1}$ (d, e, f); $t=2 \times 10^{-2} \gamma^{-1}$ (g, h, i). The other parameters are: $\zeta^{\prime} / \gamma=200, \xi^{\prime} / \gamma=100, \gamma_{2}=\gamma_{1}=\gamma$.

evidently displayed for short interaction time intervals much shorter than the characteristics relaxation time, $t \ll \gamma^{-1}$. We illustrate these effects numerically on the base of the master equation, however, in the regimes when the dissipation in the cavity is unessential and the dynamic of modes is almost unitary. For the cavity configuration presented the validity of such approximation is guaranteed by consideration of short interaction time $1 / \zeta^{\prime}, 1 / \xi^{\prime} \ll t \ll 1 / \gamma_{1,2}$ provided that the coupling constants $\zeta^{\prime}$ and $\xi^{\prime}$ exceed the dumping rates for the modes.

The reduced density operators for each of the modes are constructed from the density operator of the both modes $\rho$ by tracing over the other mode: $\rho_{1(2)}=$ $\operatorname{Tr}_{2(1)}(\rho)$. The photon number distribution for $\omega_{1}$ mode is calculated as the diagonal elements $P_{1}(n)=\left\langle n\left|\rho_{1}\right| n\right\rangle$ on the photon-number states $|n\rangle$, while calculations of the Wigner function for $\omega_{1}$ mode are performed by using its standard form in a Fock space

$$
W_{1}(\rho, \theta)=\sum_{m n} \rho_{1, m n} W_{m n}(\rho, \theta)
$$

Here: $\rho, \theta$ are the polar coordinates in the complex phase-space, which is determined by position and momentum of the quadratures $x=\left(a+a^{+}\right) / \sqrt{2}, y=(a-$ $\left.a^{+}\right) / \sqrt{2} i$, respectively, while the coefficients $W_{m n}(\rho, \theta)$ are the Fourier transforms of matrix elements of the Wigner characteristic function.

Examples of both photon-number distribution functions and the Wigner functions for $\omega_{1}$-mode are plotted in Fig. 2 for the different short time intervals. In Figs. 2 (a),(b),(c) we depict the results for $t=5 \times 10^{-4} \gamma^{-1}$. Here, the $\omega_{1}$-mode is in the vacuum state, therefore the corresponding Wigner function is Gaussian. After evolution of the system, for the time $t=5 \times 10^{-3} \gamma^{-1}$ onephoton state at the frequency $\omega_{1}$ is appeared due to the 


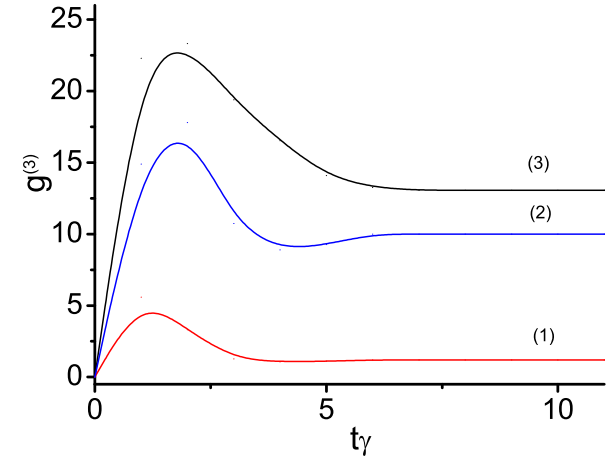

FIG. 3: Third-order correlation function versus $t \gamma$ on the threshold $E / E_{t h}=1$, curve (1); below the threshold $E / E_{t h}=$ 0.7 , curve (2) and $E / E_{t h}=0.4$, curve (3). The parameters are: $\zeta^{\prime} / \gamma=0.2, \xi^{\prime} / \gamma=0.1, \gamma_{2}=\gamma_{1}=\gamma$.

process $\omega_{0} \rightarrow \omega_{1}+\omega_{2}$, then photon triplet is generated due to the cascading $\omega_{0} \rightarrow \omega_{1}+\omega_{2} \rightarrow 3 \omega_{1}$ (see, photon distribution on the Fig. 2 (d)). The three developing arms on the Wigner function follow the semiclassical directions (see, formula (56) ) of the phase space (see, Fig. $2(\mathrm{e}),(\mathrm{f}))$. With increasing time intervals $t=2 \times 10^{-2} \gamma^{-1}$ pronounced three-photon structure of the $\omega_{1}$ mode is displayed on Fig. 2(g), (h), (i). As we see, in this case, the most probable values of photon numbers are separated by three photons. The Wigner function shows three phasecomponents with an interference pattern in the regions between them. We show the regions of quantum interference in the contour plot (see, Fig. 2(h)) depicting negative regions of the interference terms in the black. Note, that three-fold symmetry of the Wigner function and interference pattern have been demonstrated for the direct three-photon down-conversion in $\chi^{(3)}$ media [38]. However, we note that the results presented here for the cascaded configuration are different on the details from the analogous calculation on the Wigner function in [38].

Below, we consider photon-number distributions, as well as the Wigner functions of the both modes at the frequencies $\omega_{1}=\frac{\omega_{0}}{3}$ and $\omega_{2}=\frac{2 \omega_{0}}{3}$ in over-transient regime of OPO, when dissipative effect should be included.

\section{B. Photon-number correlations.}

In the paper 21] the experimental verification of timecorrelation between photons in triplet has been demonstrated. In this section, we study normalized third-order correlation function $g^{(3)}$ of the photon-numbers of mode $\omega_{1}$. This investigation is complemented by the consideration of photon number distributions of subharmonic modes. Thus, the correlation function for zero-delay time intervals is expressed as

$$
g^{(3)}(t)=\frac{\operatorname{Tr}\left[a^{+3} a^{3} \rho_{1}(t)\right]}{n_{1}^{3}(t)},
$$

where $n_{1}(t)=\operatorname{Tr}\left(a^{+} a \rho_{1}(t)\right)$ is the mean photon number. We calculate this quantity in transition near to the generation threshold of OPO in the over transient regime, $t \gg \gamma_{1}^{-1}, \gamma_{2}^{-1}$. The results of numerical calculations, on the base of the master equation (53) with the Hamiltonian (52), are shown in Fig 3 for three operation regimes of OPO. As we see, the curves (2) and (3) describe the strong correlation between three photons registered at the same time-intervals, that lead to three-photon superbunching $g^{(3)} \gg 1$. Such superbunching effect usually takes place for the direct three-photon down-conversion. We demonstrate analogous correlation for photon triplet 21] generated in the cascaded scheme. This effect decreases if the system moves to the range of generation threshold. At the threshold we have $g^{(3)}=1.2$, for $t \gamma \gg 1$ (see, curve (1), Fig. 3).

\section{Transition through the generation threshold}

We continue the analysis of the sub-section A to include dissipative effects in quantum distribution of generated mode $\omega_{1}$ and $\omega_{2}$. For this goal we concentrate on OPO operated in over transient regime, $t \gg \gamma_{1}^{-1}, \gamma_{2}^{-1}$. The results of the numerical calculations of the photonnumber distributions $P_{1}(n)$ and $P_{2}(n)$ of two modes (1) and (2) are depicted on Fig 4. As we see, below threshold (Fig 4(a)) these distributions approximately describe the statistics of spontaneous radiation. It is evident that the locations of extremum of $P(n)$-function, i.e. the locations of the most probable values of $n$, may be identified with the semiclassical stable states (54), (55) in the limit of small quantum noise level $\left(\zeta^{\prime} / \gamma_{1} \ll 1, \xi^{\prime} / \gamma_{1} \ll 1\right)$. With the increase of these ratios, due to the multiplicative character 39] of the noise in our system, the locations of these extremum becomes slightly shifted from the corresponding semiclassical values $n_{1}$ and $n_{2}$ (54), (55). As shows such comparison, the Fig 4 (c) exactly demonstrates this situation.

Another remarkable feature of cascaded OPO is formation of phase-locked states for both subharmonic modes equally separated by $2 \pi / 3$ on phase space. As follows from the semiclassical results (54), (55) there exist three states of equal intensities, but with different phases. We present below the results of numerical calculations on phase-locking in quantum theory in the framework of the Wigner functions. In below threshold regime solutions of the semiclassical equations for amplitudes of subharmonics are equal to zero, i.e. both modes are in the vacuum state, therefore the corresponding Wigner functions are almost Gaussian below threshold. The results at the threshold are shown in Fig. 5. A clear formation of phase-locked states in the transition through the generation threshold is seen. The results above the thresh- 

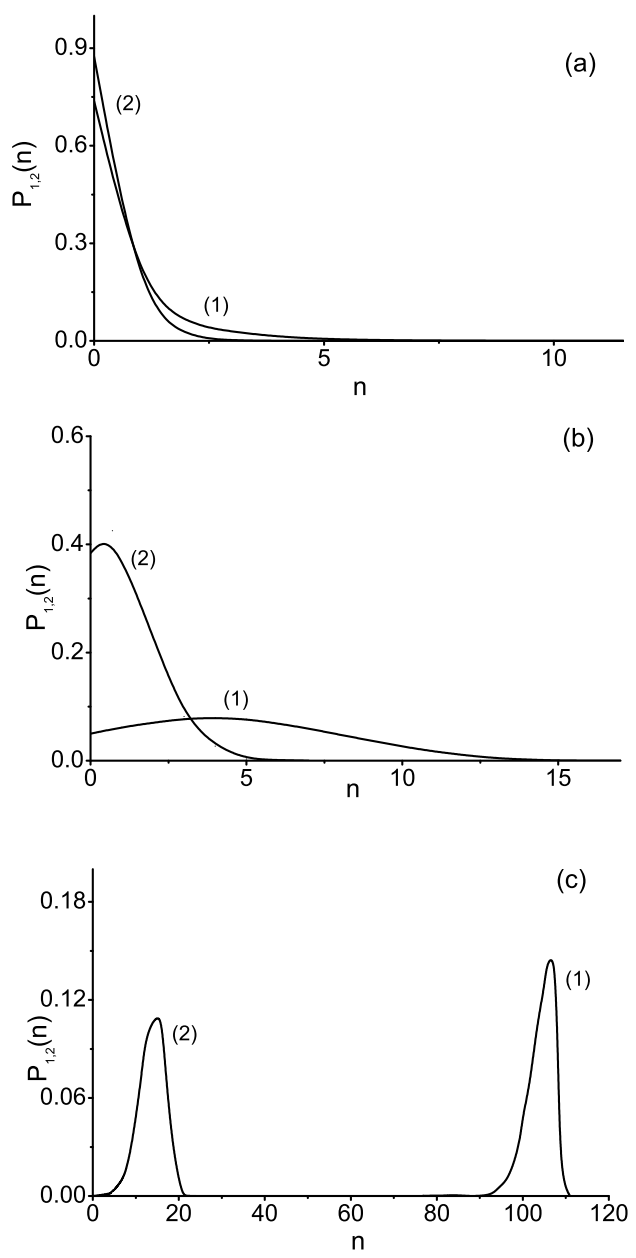

FIG. 4: Photon number distributions in transition through the generation threshold $E / E_{t h}=0.7$ for the mode (1) at the frequency $\omega_{1}=\omega_{0} / 3$ (curves (1)) and mode (2) at the frequency $\omega_{2}=2 \omega_{0} / 3$ (curves (2)). The regimes are: (a); $E / E_{t h}=1(\mathrm{~b}) ; E / E_{t h}=1.4$ (c) for the parameters: $\zeta^{\prime} / \gamma=$ $0.2, \xi^{\prime} / \gamma=0.1, \gamma_{2}=\gamma_{1}=\gamma$.

old are shown in Fig. 6, In this operational regime the Wigner functions display three-fold symmetry in phasespace in accordance with the semiclassical relations (54)(56). Note, that quantum interference pattern has been

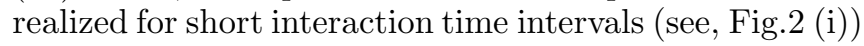
is disappeared in over transient regime in the presence of the dissipations.

\section{CONCLUSION}

In conclusion, we have investigated joint quantum state of three-photons with arbitrary spectral characteristics generated in optical superlattises. For this goal two cascaded configurations have been considered leading to production of spontaneous photon triplet in cascaded PDC

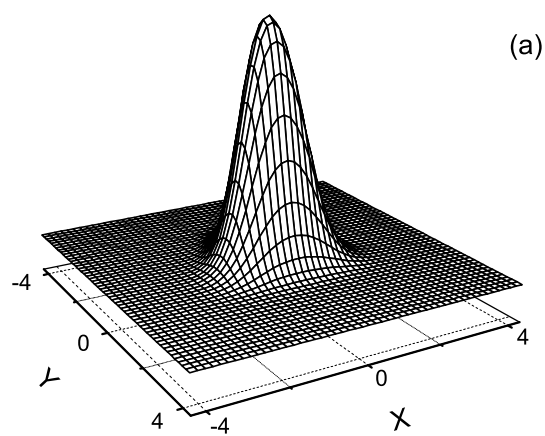

(a)

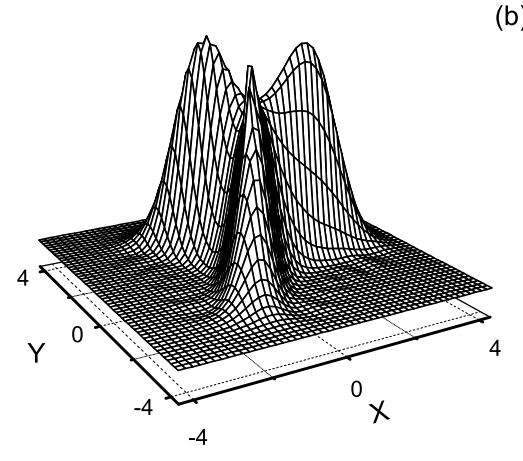

FIG. 5: Wigner functions for the modes $\omega_{2}=2 \omega_{0} / 3$ (a) and $\omega_{1}=\omega_{0} / 3(\mathrm{~b})$ at the generation threshold for the parameters: $\zeta^{\prime} / \gamma=0.2, \xi^{\prime} / \gamma=0.1, \gamma_{2}=\gamma_{1}=\gamma$ and $E / E_{t h}=1$.

and for generation of high-intensity mode through cascaded three-photon splitting in optical cavity. Considering dual-grid structure that involve periodically-poled crystals we have demonstrated that in cascaded typeII and type-I configuration triple photons constitutes GHZ entangled polarization states. We have shown that in short pulsed regime of cascaded PDC it is possible to control the three-photon joint spectra by using the method of compensation of the dispersive effects in nonlinear segments by appropriately chosen linear dispersive segment of superlattice. In the result the production of heralded joint states of two polarized photons has been demonstrated in such superlattice by using the conditional method of detection of auxiliary photon. Considering three-photon splitting in an optical cavity we have calculated photon-number distribution and the Wigner function of the mode for short interaction times. We have shown that in this regime, the Wigner function displays three-fold symmetry in phase-space (phaselocking); the three developing arms follow the semiclassical directions of the phase space with an interference pattern in the regions between them. We have also investigated three-photon cascaded down-conversion in cavity in over-transient regime when dissipative effects 
(a)
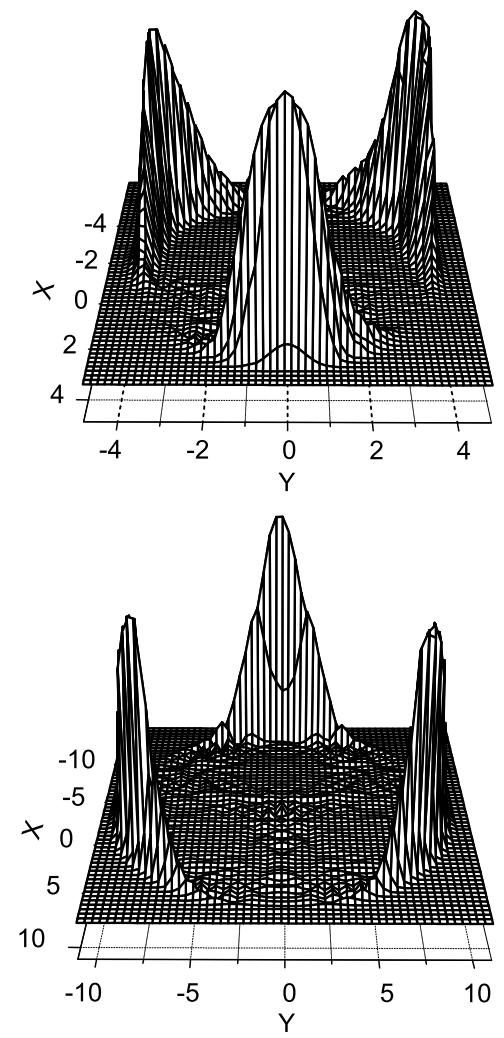

(b)
FIG. 6: Wigner functions in the operational regime above threshold $E / E_{t h}=1.4$ for the parameters: $\zeta^{\prime} / \gamma=0.2, \xi^{\prime} / \gamma=$ $0.1, \gamma_{2}=\gamma_{1}=\gamma$. (a) depicts the Wigner function for the mode $\omega_{2}$, while (b) depicts the Wigner function for the mode $\omega_{1}$. are essential. Calculating third-order correlation function of the photon-number of sub-harmonic mode we have shown the strong correlation between three photons registered at the same time-intervals that lead to three-photon super-bunching in below threshold regime. This effect decreases if the system moves to the range of generation threshold. We have also investigated the photon-number distributions and Wigner functions of sub-harmonics in transition through the generation threshold.

\section{Acknowledgments}

The research was partly founded by the ISTS (Grant No. A-1517 and CRDF/NFSAT/SCS (Grant No. ECSP09-53 A-03). We gratefully acknowledge these supports.

[1] M.A. Nielsen, I.I. Chang, ("Quantum Computation and Quantum Information", Cambridge Univ. Press), (2000).

[2] E. Knill, R. Laflamme, and G.A. Milburn, Nature 409, 46 (2001).

[3] N. Gisin, G. Ribordy, W. Tittel, and H. Zbignev, Rev. Mod. Phys. 74, 145 (2002).

[4] F. Dell'Auno, S. De Sienna, and F. Illuminati, Phys. Rep. 428, 53 (2006).

[5] D.N. Klyshko, JETP 77, 222 (1993).

[6] G. DiGiuseppe,M. Atature, M.D. Shaw, A.V. Sergienko, B.E.A. Saleh, M.C. Teich,, Phys. Rev. A 66, 013801 (2002).

[7] A.B. URen, R.K. Erdmann, M. de la Cruz-Gutierrez and I.A. Walmsley, Phys. Rev. Lett. 97, 223602 (2006).

[8] W.P. Grice, A.B. URen, and I.A. Walmsley, Phys. Rev. A 64, 063815 (2001); A.B. U'Ren, C. Silberhorn, K. Banaszek and I.A. Walmsley, Phys. Rev. Lett. 93, 093601 (2004); A.B. URen, C. Silberhorn, K. Banaszek, I.A. Walmsley, R.K. Erdmann, W.P. Grice, and M.G. Raymer, Quant.Opt. 15, 146-161 (2005).
[9] E. Bimbard, N. Join, A. MacRae, and A.I. Lvovsky, Nature Photonics 4, 242 (2010).

[10] P. Kok and S.L. Braunstein, Phys. Rev. A 62, 064301 (2000).

[11] C. Sliwa, and K. Banaszek, Phys. Rev. A 67, 030101 (2003).

[12] S. Barz, G. Cronenberg, A. Zeilinger, and P. Walther, Nature Photonics 4, 553 (2010).

[13] D.M. Greenberger, M.A. Horne, A. Shimony, and A. Zellinger, Am. J. Phys. 58, 1131 (1990).

[14] T.E. Keller, M.H. Rubin, Y. Shih, and L.A. Wu, Phys. Rev. A 57, 2076 (1998).

[15] J. Persson, T. Aichele, V. Zwiller, L. Samuelson, and O. Benson, Phys. Rev. B 69, 233314 (2004).

[16] S.N. Gupta, Phys. Rev. 96, 1453 (1954).

[17] T. Felbinger, S. Schiller, and J. Mlynek, Phys. Rev. Lett. 80,492 (1998).

[18] J. Douady, and B. Boulanger, Opt. Lett. 29, 2794 (2004).

[19] K. Bencheikh, F. Gravier, J. Douady, A. Levenson, and B. Boulanger, C.R. Phys. 8, 206 (2007). 
[20] H.C. Guo, Y.Q. Qin, and S.H. Tang, Appl. Phys. Lett. 87, 161101 (2005).

[21] H. Hübel, D.R. Hamel, A. Fedrizzi, S. Ramelow, K.J. Resch, and T. Jennewein, Nature Photonics Lett. 466, 601 (2010).

[22] K.J. Resch, Ph. Walther, A. Zeilinger, Phys. Rev. Lett. 94(7), 070402 (2005).

[23] J.M. Wen and M. H. Rubin, Phys. Rev. A 79, 025802 (2009).

[24] J.M. Wen, E. Oh, and Sh. Du, J. Opt. Soc. Am. B, Opt. Phys. 27(6), A11 (2010).

[25] G.Yu. Kryuchkyan, N.T. Muradyan, Phys. Lett., A 286, 113 (2001).

[26] J.J. Zondy, A. Tallet, E. Ressayre, and M. LeBerre, Phys. Rev. A 63, 023814 (2001).

[27] G.Yu. Kryuchkyan, L.A. Manukyan and N.T. Muradyan, Opt. Comm. 190, 245 (2001).

[28] J.M. Wen, P. Xu, M. H. Rubin, and Y.H. Shih, Phys. Rev. A 76, 023828 (2007).

[29] D.A. Antonosyan, and G.Yu. Kryuchkyan, ("Modern Optics and Photonics: Atoms and Structured Media" World
Scientific Publishing Co.,131-150, 2010).

[30] P. Leung, T. Ralph, W. J. Munro, and K. Nemoto, arXiv:quant-ph:0810.2828v2 (2008).

[31] P.M. Leung, W.J. Munro, K. Nemoto, and T.C. Ralph, Phys. Rev. A 79, 042307 (2009).

[32] P.V. Gorelik, F.N.C. Wong, D. Kolker, and J.J. Zondy, Opt. Lett. 31, 2039 (2006).

[33] P.G. Kwiat, K. Mattle, H. Weinfurter, A. Zeilinger, A.V. Sergienko and Y. Shih, Phys. Rev. Lett. 75, 4337 (1995).

[34] T.E. Keller and M.H. Rubin, Phys. Rev. A 56, 1534 (1997).

[35] N. Gisin and I.C. Percival, J. Phys. A 26, 2233 (1993);N. Gisin and I.C. Percival, J. Phys. A 26, 2245 (1993).

[36] H.H. Adamyan, S.B. Manvelyan, G.Yu. Kryuchkyan, Phys. Rev. A 63, 022102 (2001).

[37] H.H. Adamyan, S.B. Manvelyan, G.Yu. Kryuchkyan, Phys. Rev. E 64, 046219 (2001).

[38] K.Banaszek and P.Knight, Phys. Rev. A 55, 2368 (1997).

[39] W. Horsthemke and R. Lefever, Nois-Induced Transitions (Springer, Berlin, 1984). 